\title{
Group I metabotropic glutamate receptors activate burst firing in rat midbrain dopaminergic neurons
}

\author{
Simonetta Prisco a , Silvia Natoli a , Giorgio Bernardi a, b, Nicola B. Mercuri a, b,* \\ ${ }^{a}$ IRCCS Fondazione S. Lucia, Experimental Neurology Laboratory, Via Ardeatina 306, 00179 Rome, Italy \\ ${ }^{\mathrm{b}}$ Clinica Neurologica, Università di Tor Vergata, Rome, Italy
}

Received 31 May 2001; received in revised form 5 November 2001; accepted 4 December 2001

\begin{abstract}
We have investigated the changes in the spontaneous firing pattern induced by DHPG ((S)-3,5-dihydroxyphenylglycine) and NMDA (N-methyl-d-aspartic acid) on rat dopaminergic neurons in substantia nigra pars compacta (SNc) using sharp microelectrode recordings in in vitro conditions. Twenty-five out of 33 cells modified the regular single-pacemaker activity in burst firing when exposed to the Group I metabotropic glutamate receptor (mGluR) agonist DHPG $(30 \mu \mathrm{M})$ and d-tubocurarine $(500 \mu \mathrm{M})(\mathrm{d}-\mathrm{TC})$, whereas they all fired in bursts during NMDA $(20 \mu \mathrm{M})$ plus d-TC application. The blockade of SK-channels by d-TC and apamin was essential for the production of both types of bursts. Although the two drugs induced a similar number of action potentials per burst, the DHPG-induced bursts had a lower frequency, a longer duration and a longer plateau period without spikes. In addition, the DHPG-induced bursting had a longer wash-out, could be reduced or blocked by the mGluR 1 selective, non-competitive antagonist CPCCOEt (7-cyclopropan[b]chromen-1a-carboxylic acid ethyl ester) $(100 \mu \mathrm{M})$ while it was not affected by the mGluR 5 selective antagonist MPEP (2-methyl-6-(phenylethynyl)-pyridine $(10 \mu \mathrm{M})$. These results suggest that both the activation of glutamate metabotropic type 1 and NMDA ionotropic receptors induce burst firing in the dopaminergic cells of the ventral midbrain when the activity of the SK-channels is reduced. (C) 2002 Elsevier Science Ltd. All rights reserved.
\end{abstract}

Keywords: DHPG; NMDA; d-tubocurarine; Apamin; SK-channels; Single-spike mode; Bursts; Dopaminergic neurons

\section{Introduction}

The discharging pattern of midbrain dopaminergic cells has a profound influence on the overall functioning of the dopamine system. Several studies have already shown that dopaminergic neurons display different mode of activity when recorded in in vivo conditions. In particular, they fire either burst of action potentials or irregular single spikes (Bunney et al., 1973; Grace and Bunney, 1984a,b; Grace and Onn, 1989; Shepard and German, 1988; Wilson et al., 1977). Interestingly, the bursting activity maximizes the release of dopamine (DA) in the brain (Overton and Clark, 1997). In fact, the amount of extracellular DA is enhanced in the striatum, nucleus accumbens and cerebral cortex when dopaminergic neurons switch from a single-spike firing to bursts

* Corresponding author. Tel.: +39-06-51501383; fax: +39-0651501384.

E-mail address: mercurin@med.uniroma2.it (N.B. Mercuri). of action potentials (Gonon, 1988; Gonon and Buda, 1985; Manley et al., 1992; Nissbrandt et al., 1994). This bursting activation is also related to relevant and novel rewarding stimuli (Freeman et al., 1985; Horvitz et al., 1997; Mirenowicz and Schultz, 1996; Schultz, 1998).

The dopaminergic neurons, when recorded in in vitro conditions, are mainly characterized by a regular, singlespike spontaneous firing (Grace and Onn, 1989; Johnson and North, 1992; Lacey et al., 1989; Yung et al., 1991). Thus, the absence of the variety of activity patterns observed in vivo is presumably due to the loss of extrinsic afferents impinging on these neurons. The particular importance of excitatory synaptic inputs has been suggested by the observation that: (a) the stimulation of $\mathrm{N}$ methyl-d-aspartic acid (NMDA)-receptors plays an essential role in the discharge pattern of these cells in in vivo experiments; (Chergui et al., 1993; Tong et al., 1996) (b) the continuous activation of NMDA receptors, when associated with modifications of intrinsic membrane components (Kitai et al., 1999), transforms the typical pacemaker-like discharge of the dopaminergic 
neurons in a bursting mode in in vitro conditions (Johnson et al., 1992; Wang et al., 1994). However, the tonic activation of ionotropic receptors of the NMDA type may not be the exclusive determinant of the bursting behavior of these cells. For instance, the activation of metabotropic glutamate receptors (mGluRs) could be also involved. Therefore, the purpose of the present study was to determine whether group I mGluRs agonists, that exert an excitatory effect on dopaminergic neurons in in vitro experiments (Mercuri et al., 1993) and increase the frequency of bursts in in vivo conditions (Meltzer et al., 1997), could induce, under particular conditions, bursting activity in these cells. To test for this possibility, in vitro intracellular recording techniques were used and the selective group I mGluR agonist (S)-3,5-dihydroxyphenylglycine (DHPG) (Conn and Pin, 1997; Shoepp et al., 1994) was applied, in the presence of tubocurarine (d-TC) or apamin, on the dopaminergic cells of the substantia nigra pars compacta $(\mathrm{SNc})$. Tubocurarine at a high concentration $(500 \mu \mathrm{M})$, has reversible apamin-like effects, blocking a small $\mathrm{Ca}^{++}$ activated $\mathrm{K}^{+}$current $(\mathrm{SK})$ and reducing the amplitude of the post-spike afterhyperpolarization (AHP) (Brodie et al., 1999; Ishii et al., 1997; Köhler et al., 1996).

\section{Methods}

\subsection{Preparation of the tissue}

The method used has been described previously (Mercuri et al., 1995). In brief, Wistar rats (150-250 g) were anaesthetized with ketamine and killed. All efforts were made to minimize animal suffering and the number of animals used. All experiments follow international as well as local guidelines on the ethical use of animals from the European Communities Council Directive of 24 November 1986 (86/609/EEC) and the ethical committee of the University of Tor Vergata (Rome, Italy). The brain was removed and horizontal slices (thickness $300 \mu \mathrm{m})$ were cut by a vibratome starting from the ventral surface of the midbrain. A single slice containing the substantia nigra pars compacta was then transferred into a recording chamber and completely submerged in an artificial cerebrospinal fluid with a continuously flowing $(2.5 \mathrm{ml} / \mathrm{min})$ solution at $35{ }^{\circ} \mathrm{C},(\mathrm{pH} \mathrm{7.4})$. This solution contained $(\mathrm{mM}): \mathrm{NaCl} 126 ; \mathrm{KCl} 2.5 ; \mathrm{MgCl}_{2}$ 1.2; $\mathrm{NaH}_{2} \mathrm{PO}_{4} 1.2 ; \mathrm{CaCl}_{2} 2.4 ;$ Glucose 11, $\mathrm{NaHCO}_{3} 20$; gassed with $95 \% \mathrm{O}_{2}$ and $5 \% \mathrm{CO}_{2}$.

\subsection{Electrophysiology}

Intracellular recording electrodes (Clark 1-1.5 mm, thick wall), pulled by Narishige and Flaming-Brown horizontal pullers, were filled with $2 \mathrm{M} \mathrm{KCl}$ yielding a DC resistance of 40-80 M $\Omega$. The tips of the electrodes were placed in the SNc by using a dissecting microscope. The signals were obtained by an amplifier (Axoclamp-2, Axon Instruments) in continuous current clamp (bridge) mode, displayed on a pen recorder (Gould $2400 \mathrm{~S}$ ) and digitized using a digidata interface driven by Axotape (Axon Instruments) running on an AT-compatible computer and saved for off-line analysis.

\subsection{Application of drugs}

Drugs were made in stock solutions and bath applied at known concentrations via a three-way tap system. A complete exchange of the solution in the recording chamber occurred in approx. 1 min. (S)-3,5-dihydroxyphenylglycine (DHPG), 2-methyl-6-(phenylethynyl)pyridine (MPEP) and 7-cyclopropan[b]chromen-1a-carboxylic acid ethyl ester (CPCCOEt) were obtained from Tocris Cookson (Bristol, UK); N-methyl-d-aspartic acid (NMDA), d-tubocurarine (d-TC) and dopamine (DA) were from Sigma Chemical Company (Milan, Italy); apamin from Alomone lab (Jerusalem, Israel).

\subsection{Statistics and burst characterization}

The changes in firing rate induced by the drugs (during 3 min of application) were normalized as a percentage of control (each neuron served as its own control). Using a software program developed in our laboratory and running on an IBM computer we performed spike and burst analysis. The detection of an interspike of $300 \mathrm{~ms}$ or less was considered to represent the onset of a burst (composed of three or more action potentials). A burst of spikes is superimposed on a depolarizing phase of the membrane potential that progresses to and terminates when the cell membrane is driven into a temporary state of depolarization block (Fig. 1) (Shepard and Bunney, 1991). Thus, a plateau potential comprises a period of higher firing discharge followed by spike

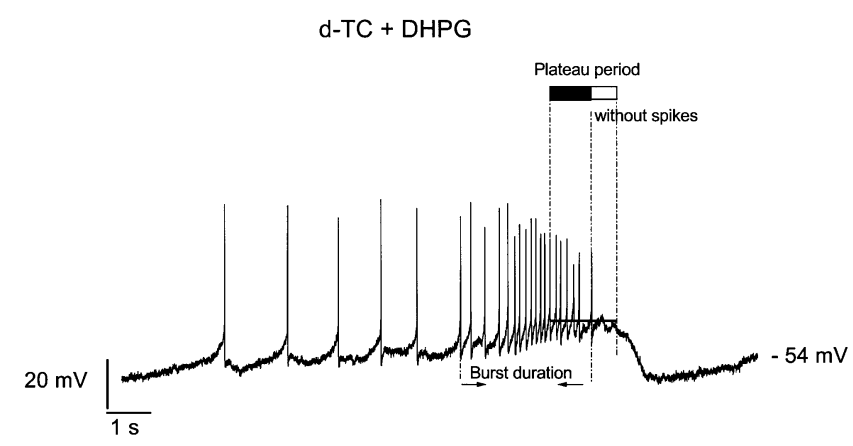

Fig. 1. Characteristics of the DHPG-induced burst. The trace shows a burst, indicated by the two arrows, caused by the application of DHPG $(30 \mu \mathrm{M})$ and d-TC $(500 \mu \mathrm{M})$ on a dopaminergic cell. Note the action potentials superimposed on a depolarizing envelope followed by spike inactivation. The bar indicates the plateau period with spikes (black) and without spikes (white). 
inactivation (plateau period without spikes). A post-plateau membrane hyperpolarization terminated the plateau potential. Data were expressed as mean \pm standard error of the mean. Differences in data were evaluated by Student's $t$ test and were considered significant when $P<$ 0.05 .

\section{Results}

\subsection{Properties of the cells}

The data presented below were obtained from presumed 'principal' dopaminergic neurons of the substantia nigra pars compacta. These cells fired spontaneously in a pacemaker-like mode [Fig. 2(a)] at a rate of $1.2 \pm 0.3$
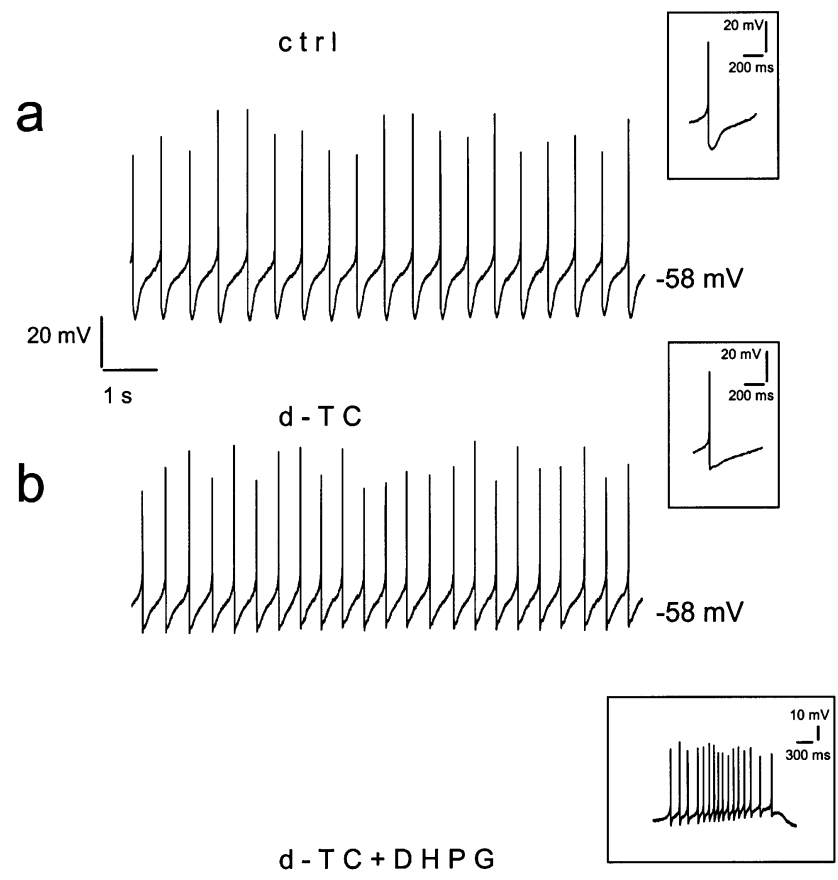

C

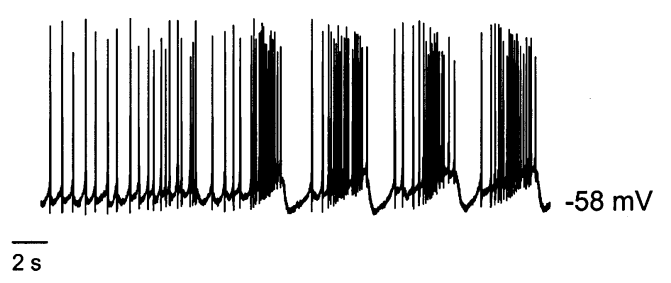

Fig. 2. Effects of DHPG and d-TC on the firing pattern of the dopaminergic cells. (a) The trace shows intracellular voltage recording of a dopaminergic cell spontaneously firing in a regular pacemaker-like mode. Due to the limited capacity of the digitizing processes, in this and the following figures the full amplitude of the spikes is not reproduced. An individual action potential is shown in the inset (note the pronounced afterhyperpolarization). (b) d-TC $(500 \mu \mathrm{M})$, during $6 \mathrm{~min}$ of treatment, causes an increase in firing rate but does not modify the firing pattern. The inset shows an individual spike. Note the depression of the AHP. (c) The superfusion of DHPG (30 $\mu \mathrm{M}, 4-6$ min) induces the activation of a bursting discharge in this d-TC-treated cell. An individual burst is shown in the inset.
$\mathrm{Hz}(n=34)$ and presented action potentials longer than $1.4 \mathrm{~ms}$. The mean interspike interval (ISI) was $860.3 \pm 65.5 \mathrm{~ms}(n=34)$. They also displayed a pronounced time and voltage-dependent rectification $I_{\mathrm{h}}$, during hyperpolarizing voltage pulses. In addition, the application of DA $(10-30 \mu \mathrm{M})$ caused a hyperpolarizing response (5-13 $\mathrm{mV}, n=15$, not shown). These characteristics have been shown to be specific for midbrain dopaminergic neurons recorded in vitro (Grace and Onn, 1989; Kita et al., 1986; Lacey et al., 1989; Mercuri et al., 1995).

\subsection{Effects of $d$-tubocurarine on the firing rate}

Bath-applied d-TC $(500 \mu \mathrm{M})$ increased the spontaneous action potential firing rate of dopaminergic neurons by $22.6 \pm 3.9 \%$ of control $(n=33$; Student's $t$ test, $P<0.01)$, but it did not induce any change in firing pattern [Fig. 2(b) and 3(a)]. The mean ISI of dopaminergic cells in the presence of d-TC was 555.4 \pm 23.9 ( $n=33$ ).

Although the interspike interval remained regular, application of d-TC reduced the amplitude and the duration of the AHP that follows the action potentials (it mainly reduced the slow component, inset of Fig. 2(b)]. Under tubocurarine treatment, the intracellular injection of a hyperpolarizing current reduced or blocked the firing discharge while the injection of a sustained depolarizing current increased the spontaneous firing rate without changing its pattern (not shown).

\subsection{Effects of (S)-3,5-dihydroxyphenylglycine on the activity of dopaminergic cells}

The superfusion of DHPG $(30 \mu \mathrm{M})$ for $4-6 \mathrm{~min}$ in the presence of d-TC (6 min pretreatment) induced a continuous bursting[Fig. 2(c)] in the majority (25/33) of tested cells. Regular plateau potentials were surmounted by bursts of smaller and wider spikes that were often inactivated. The plateau was then terminated by a repolarization (Fig. 1). In some cells $(n=8)$ the depolarizing plateau developed but no spikes were present on it (not shown). After DHPG wash-out the spontaneous activity slowly recovered to single-spike mode (between 8 and $12 \mathrm{~min}$, Fig. 3(b)]. In the remaining neurons, the group I mGluRs agonist, added to the perfusate containing dTC, increased the firing rate by $143.3 \% \pm 18.7$ of control (Student's $t$ test, $P<0.01$; ISI=270.1 \pm 22.3 ) without changing the discharge pattern [Fig. 3(a)].

In addition, a series of experiments were carried out to test whether DHPG in the absence of d-TC, could cause burst firing. When applied to the bathing solution DHPG $(30 \mu \mathrm{M})$ significantly increased the basal firing rate of dopaminergic cells $(n=12$; mean increase over base line $135.0 \% \pm 6.3$; Student's $t$ test, $P<0.05$; ISI $=290 \pm 16.3$ ), but it did not induce any bursting 
a

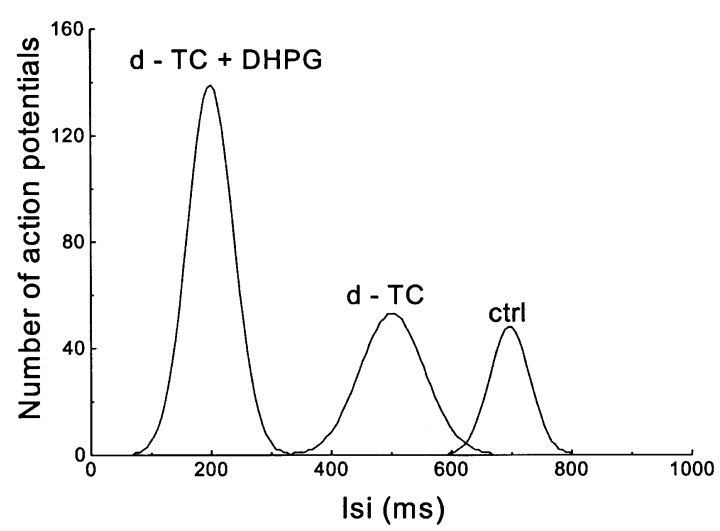

b

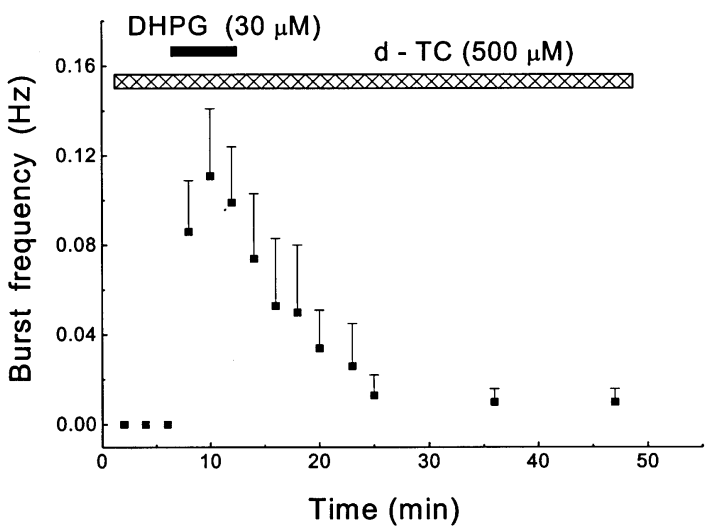

Fig. 3. Effect of d-TC and d-TC plus DHPG on the dopaminergic cells. (a) The three curves describe the distribution of the ISI in control condition (ctrl), in d-TC $(500 \mu \mathrm{M})$ and during the addition of DHPG $(30 \mu \mathrm{M})$ in a dopaminergic cell that responds with a linear increase in firing rate to the application of the drugs. These curves are obtained by the superimposition of Gaussian distribution approximating the experimental ISI histograms. Each Gaussian is described by this equation: $f(x)=1 /\left((2 \pi)^{1 / 2} \sigma\right)^{\left(-(X-\mu)^{2} / 2 \sigma^{2}\right)}$ where $\mu$ is the mean interspike interval and $\sigma$ is the mean standard deviation. Note the leftward shift of the ISI distribution induced by d-TC and d-TC plus DHPG, indicating a strong increase in the firing rate (time interval of acquisition $\Delta x=40 \mathrm{~s}$; bin width=100 ms). (b) Time course of the bursting frequency caused by DHPG in d-TC-treated neurons $(n=15)$. Note the relative long period of time required for wash-out.

behavior [Fig. 4(b)]. Thus, it appears that a simultaneous reduction of the slow afterhyperpolarization (AHP) with d-TC and activation of group I mGluRs are necessary to induce a bursting pattern in midbrain dopaminergic cells.

Furthermore, a pretreatment of the cells with apamin a more selective blocker of SK channel (6 min, $100 \mathrm{nM})$ caused a linear increase in firing rate $(n=4 ; 12.3 \% \pm 2.5$; Student's $t$ test, $P>0.05$; ISI $=662.3 \pm 27.0)$ and a clear reduction of the AHP [Fig. 5(b)] but it did not modify the firing pattern. The subsequent addition of DHPG (30 $\mu \mathrm{M}$ ) caused a burst firing in 2 out of 4 neurons [Fig. $5(\mathrm{c})]$.

\section{4. mGluR 1 mediate the (S)-3,5- dihydroxyphenylglycine-induced bursting}

In a group of cells we tested whether the DHPGinduced bursting was sensitive to glutamate metabotropic receptor blockade. CPCCOEt (a selective noncompetitive mGluR1 antagonist) (Casabona et al., 1997) $(100 \mu \mathrm{M})$ antagonized in $8-13 \mathrm{~min}$ the DHPG/d-TCinduced burst firing in the dopaminergic cells $(n=3$; Fig. 6). On the other hand, the superfusion of the mGluR 5 selective antagonist MPEP (2-methyl-6(phenylethynyl)-pyridine) (10 $\mu \mathrm{M})$ (Gasparini et al., 1999) did not affect the DHPG-induced bursting in three cells (not shown).

\subsection{Both (S)-3,5-dihydroxyphenylglycine or N-methyl- $d$-aspartic acid evoke bursting in dopaminergic cells}

The dopaminergic neurons treated with NMDA (20 $\mu \mathrm{M})$ and $\mathrm{d}-\mathrm{TC}$ all generated a pronounced bursting activity. Interestingly, either NMDA or DHPG could induce bursting pattern in the presence of d-TC (Fig. 7). When NMDA (top) or DHPG (bottom) were added to the superfusate containing d-TC, the representative ISIs showed a marked switch to a burst-firing mode [Fig. 7(a,b)]. The DHPG- and NMDA-induced bursting activity could be reduced or stopped by a hyperpolarization of the membrane below $-65 \mathrm{mV}$ (not shown). The characteristics of the bursts induced by the two drugs are shown in Table 1. The number of bursts, burst duration, the period of the plateau without spikes and washout were different for the two classes of drugs. However, the mean number of spikes per burst was almost similar during DHPG or NMDA application.

It should be also mentioned that a constant hyperpolarizing current injection $(-30,-120 \mathrm{pA})$ was required to facilitate the NMDA-induced bursts (Johnson et al., 1992) while no hyperpolarizing current had to be injected into the cells during the effects of DHPG to sustain membrane oscillations. Finally, we also searched for a possible synergistic action of DHPG and NMDA in the induction of bursts. When applied to the bathing solution containing no d-TC, DHPG $(30 \mu \mathrm{M})$ plus NMDA $(30 \mu \mathrm{M})$ depolarized the membrane and increased the firing rate that inactivated. The intracellular injection of a hyperpolarizing current reestablished linear firing and did not induce bursts [Fig. 4(c)] $(n=4)$ when the membrane was held between -35 and -57 $\mathrm{mV}$.

\section{Discussion}

The present in vitro study shows that the activation of group I mGluRs, as well as NMDA receptors, can 
ctrl

a

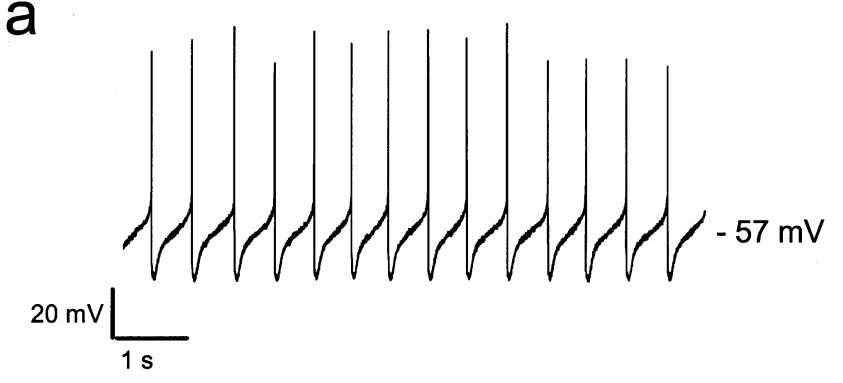

b

DHPG

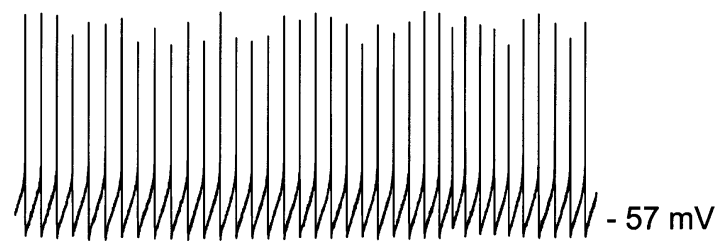

DHPG + NMDA

C

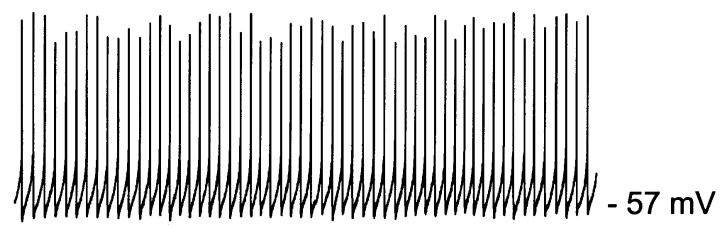

Fig. 4. DHPG in the absence of d-TC plus NMDA is unable to induce burst firing in dopaminergic neurons. (a) Control: the trace shows individual spontaneous action potentials. (b) DHPG (30 $\mu \mathrm{M})$ generates a linear increase in firing activity. (c) DHPG plus NMDA $(20 \mu \mathrm{M}) \mathrm{did}$ not change firing pattern. Note that, during the effect of NMDA, 180 $\mathrm{pA}$ of hyperpolarizing current had to be injected in order to block an excessive membrane depolarization.

induce a switch of the spontaneous firing activity from a single-spike to a bursting mode in midbrain dopaminergic neurons. These data are in line with the current hypothesis that the glutamatergic synaptic inputs activating NMDA receptors control the firing pattern of dopaminergic neurons (Johnson et al., 1992; Overton and Clark, 1997; Wang et al., 1994). In addition, they also suggest a new role of mGluRs in regulating the firing pattern. Considering that long-lasting excitatory events (Shen and Johnson, 1997) are caused by group I mGluRs synaptic stimulation, it is intuitive to speculate that these excitatory inputs regulate the depolarizing drive sustaining the burst in the dopaminergic cells. On the other hand, the inhibitory potentials induced by mGluR1 activation have been shown to be highly desensitizing (Fiorillo et al., 1998) therefore it is unclear what their role in bursting activity might be. This remains to be explored in future experiments. An increase of bursting

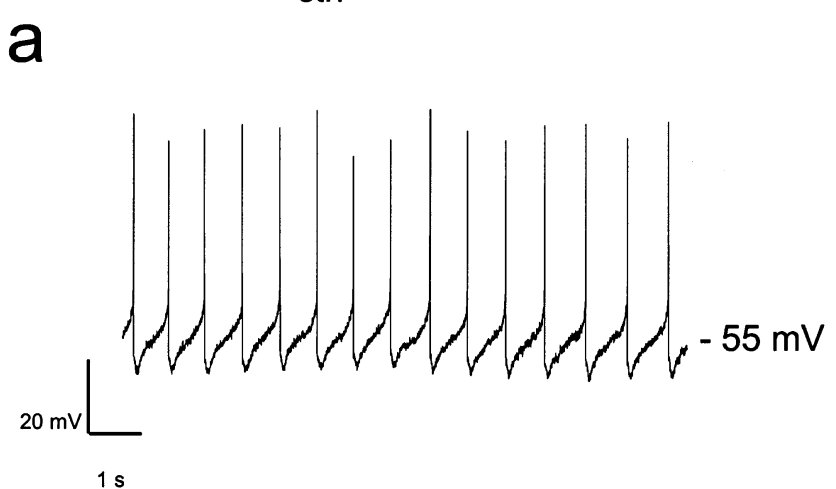

apamin

b
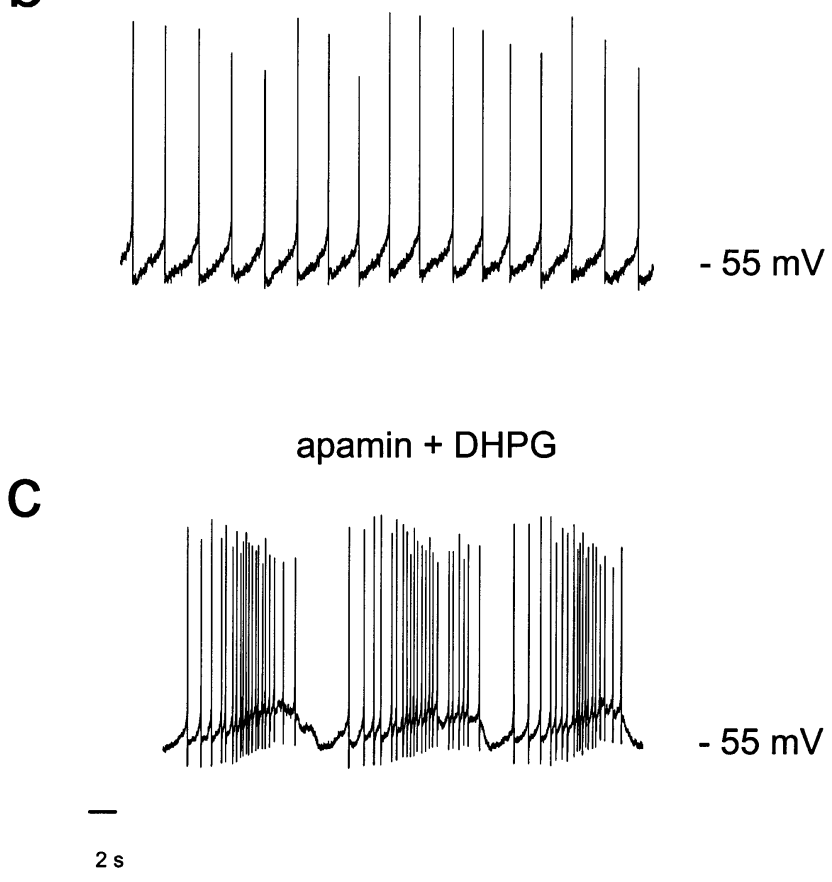

Fig. 5. Apamin enables the DHPG-induced bursts. (a) The trace shows intracellular voltage recording of a dopaminergic cell spontaneously firing in a regular pacemaker-like mode. (b) apamin (100 $\mathrm{nM}$ ), during 6 min of treatment, causes a small increase in firing rate but does not modify the firing pattern. (c) The superfusion of DHPG (30 $\mu \mathrm{M}, 4-6 \mathrm{~min}$ ) induces the activation of a bursting discharge in this apamin-treated cell.

frequency is also caused by the application of the group I-II agonist trans-D,L-1-amino-1,3-cyclopentanedicarboxylic acid ( $\mathrm{t}-A C P D)$ on the dopaminergic neurons in in vivo preparations (Meltzer et al., 1997). Interestingly, mGluRs activation has been shown to induce membrane potential oscillations and bursting in dorsal septal neurons (Zheng and Gallagher, 1991).

In agreement with the recent in vitro data of Brodie et al. (1999), we have also reported that a treatment with d-TC causes a small but significant increase in the firing rate of dopaminergic neurons while it does not induce burst pattern. Similar effects were also caused by apa- 
a ctrl

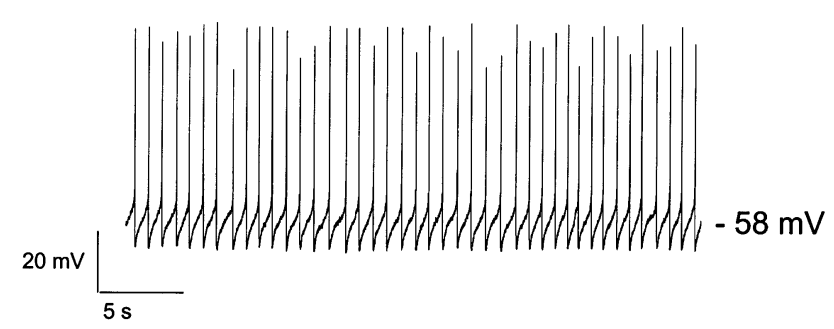

b

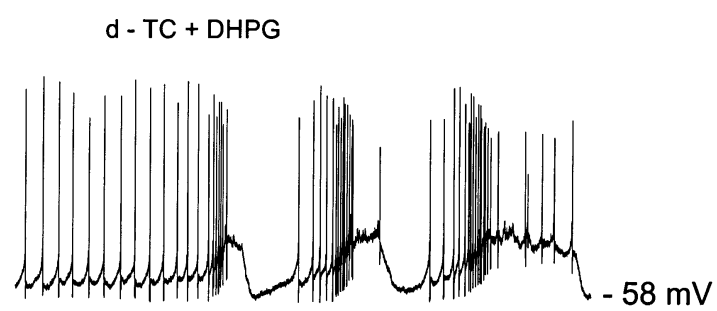

C

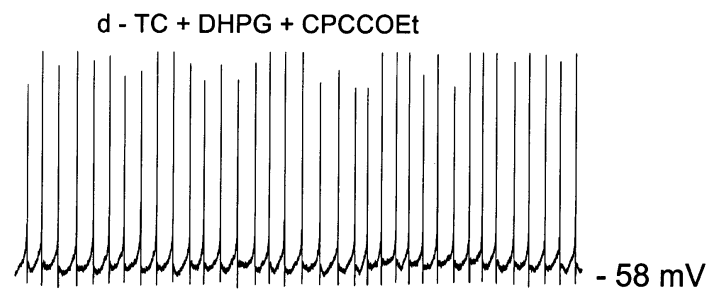

Fig. 6. Blockade the DHPG-induced bursts by CPCCOEt in dopaminergic cells. (a) The trace shows voltage recording of a spontaneously firing dopaminergic cell in control condition (ctrl). (b) The subsequent application of DHPG $(30 \mu \mathrm{M})$ induces burst firing in this cell pretreated with d-TC (500 $\mu \mathrm{M}$, for $6 \mathrm{~min})$. (c) The addition of the mGluR 1 antagonist, CPCCOEt (100 $\mu \mathrm{M}$, for $10 \mathrm{~min}$ ), abolishes burst firing in spite of the continuous treatment with d-TC plus DHPG.

min, a more potent and selective antagonist of SK channels. Therefore, the reduction of SK current by high concentration of d-TC or apamin is an essential prerequisite for the DHPG-induced bursts in in vitro conditions. This is consistent with previous studies (Seutin et al., 1993) indicating that apamin facilitates the NMDA-induced bursting activity in dopaminergic cells. The possible existence of neurotransmitters modulating the SK current has been already postulated (Kitai et al., 1999), in order to account for the different pattern of firing in the dopaminergic cells. Remarkably, in a recent paper Paladini et al. (2001) have demonstrated that $\alpha 1$ receptors stimulation reduces an SK-dependent hyperpolarization and this could certainly participate to the mGluR1induced burst firing. In addition, as already reported by Brodie et al. (1999) the ethanol-induced reduction of the post-spike AHP in the dopaminergic cells could facilitate burst firing when associated to mGluRs stimulation. Moreover, the observations that a simple depolarization a

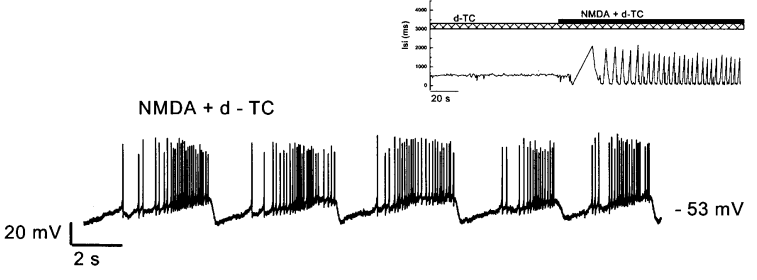

b

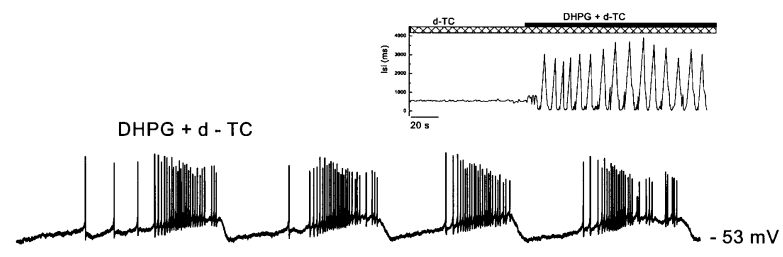

Fig. 7. Both NMDA and DHPG induce bursting activity on d-TC treated neurons. (a) Top: time course of the changes of the ISI along with time caused by NMDA in the cell shown below. Before application of the drugs to a Ringer solution containing d-TC, the neuron fired in a pacemaker-like pattern as indicated by the linearity of interspike intervals. Note the presence of a large peak evoked by an initial condition of NMDA-application+injection of hyperpolarizing current until when a bursting cycle was reached. Bottom: the trace shows regular bursts in the presence of NMDA $(20 \mu \mathrm{M})$. (b) Top: time course of the changes of the ISI along time caused by DHPG in the same cell shown in a. Before application of the drug to a Ringer solution containing d-TC and after the wash-out of NMDA, the neuron fired in a pacemaker-like pattern as indicated by the linearity of interspike intervals. Note the different distribution of the ISIs among the NMDA- and DHPG-induced bursts. Bottom: the trace shows regularly occurring bursts in the presence of DHPG $(30 \mu \mathrm{M})$.

Table 1

Characteristics of the bursts ${ }^{\mathrm{a}}$

\begin{tabular}{|c|c|c|}
\hline & $\begin{array}{l}\text { DHPG }+ \text { dTC } \\
(n=9)\end{array}$ & $\begin{array}{l}\text { NMDA+d-TC } \\
(n=11)\end{array}$ \\
\hline Bursts $\left(\min ^{-1}\right)$ & $7.38 \pm 2.70 *$ & $46.59 \pm 15.00$ \\
\hline Burst duration (s) & $3.72 \pm 0.14 *$ & $0.10 \pm 0.04$ \\
\hline Plateau period without Spikes (s) & $0.34 \pm 0.14 *$ & $0.10 \pm 0.04$ \\
\hline Spikes per burst & $16.00 \pm 6.00$ & $17.10 \pm 6.00$ \\
\hline Wash-out & $10.80 \pm 2.40 *$ & $2.04 \pm 0.15$ \\
\hline
\end{tabular}

a The dopaminergic cells were treated for $6 \mathrm{~min}$ with d-TC and subsequently exposed to NMDA $(20 \mu \mathrm{M})$ or DHPG $(30 \mu \mathrm{M})$. All the values were calculated during the first $3 \mathrm{~min}$ of burst induction, The asterisks indicate significant differences between values (Student' $t$ test, $P<0.01)$. During the effects of DHPG the membrane potential oscillated between -57 and $-35 \mathrm{mV}$. This range of membrane potential was also maintained by current injection during the NMDAinduced bursts.

of the cell membrane is not sufficient to induce bursting even when the SK channels are blocked and the DHPGand NMDA-induced bursting are blocked by hyperpolarization suggest that a receptor-activated voltage-dependent negative slope conductance (Guatteo et al., 1999 Mercuri et al. 1992, 1993; Wu and Johnson, 1996) sustains the bursting behaviour. On the basis of the antagonism obtained with CPCCOEt and the lack of effects of MPEP (Tozzi et al., 2001) is very likely that the receptor 
subtype involved in the DHPG-induced bursting is the mGluR1, whose activation induces an inward current mainly carried by sodium ions in the dopaminergic cells (Guatteo et al., 1999; Mercuri et al., 1993). The slow time course of the effects of CPCCOEt suggests that the prolonged wash-out of DHPG might depend on the persistent intracellular metabolic events induced by the agonist rather than a sustained occupancy of the mGluRs. On the basis of the distinct characteristics of the DHPG- and NMDA-induced bursts we suggest a peculiar role for the group I mGluRs. It is interesting to note that: (a) DHPG, but not NMDA, leads to prolonged changes in the firing pattern of dopaminergic neurons which can persist for several minutes after the end of the perfusion of the drug; (b) DHPG-burst does not require continuous hyperpolarizing current injection to develop. This might depend on the fact that the depolarizing drive caused by DHPG does not move the membrane potential out a critical window necessary for the development of voltage-dependent plateau potentials (Kitai et al., 1999). Thus, unless hyperpolarized by intracellular current injection we were not able to evoke NMDA-induced burst firing because the cells went in depolarization block. This might have a significance in the regulation the dopaminergic system rendering the NMDA-induced stimulus transient because inactivating. On the other hand, the oscillatory activity generated by a mGluR 1 stimulation can be an enduring signal having a longer time course than the NMDA-induced one.

Thus, both types of bursts may have importance in the information coding which results from glutamate transmission on dopaminergic neurons. The resultant changes in the temporal organization of neuronal spike trains within the bursts might represent different mechanisms used by the dopaminergic neurons to alter their influence on target cells in the forebrain.

It is also interesting to note that the simultaneous activation of ionotropic NMDA receptors and group I mGluRs does not induce bursting again suggesting that the blockade of SK channels is an important requirement for burst generation.

\section{Conclusions}

The present study provides the first evidence for a role of mGluRs 1 in the activation of bursting behavior in midbrain DA-containing neurons. This effect is observed in the majority of cells exposed to the mGluR 1 agonist DHPG and require the closure of the SK channels. Since bursts are more effective than single-spike firing to increase DA release in the brain, the involvement of metabotropic glutamate receptors in this phenomenon further strengthen the role of this amino acid in transforming the tonic release of the catecholamine in phasic release. Interestingly, a modification in bursting activity might have importance in the sensitization to drugs of abuse (Vezina and Kim, 1999) and could cause disorders (schizophrenia and drug dependence), which are linked to a deranged function of both DA and glutamate Carlsson and Carlsson, 1990).

\section{Acknowledgements}

The technical assistance of M. Federici is gratefully acknowledged.

\section{References}

Brodie, M.S., McElvain, M.A., Bunney, E.B., Appel, S.B., 1999. Pharmacological reduction of small conductance calcium-activated potassium current (SK) potentiates the excitatory effect of ethanol on ventral tegmental area dopamine neurons. Journal of Pharmacology and Experimental Therapeutics 290, 325-333.

Bunney, B.S., Walters, J.W., Roth, R.H., Aghajanian, G.K., 1973. Dopaminergic neurons effect of antipsychotic drugs and amphetamine on single cell activity. Journal of Pharmacology and Experimental Therapeutics 185, 560-571.

Carlsson, M., Carlsson, A., 1990. Interaction between glutamatergic and monoaminergic system within the basal ganglia-implications for schizophrenia and Parkinson's disease. Trends Pharmacological Science 13, 272-276.

Casabona, G., Knopfel, T., Kuhn, R., Gasparini, F., Baumann, P., Sortino, M.A., et al. 1997. Expression and coupling to phosphoinositide hydrolysis of group I metabotropic glutamate receptors in early postnatal and adult rat brain. European Journal of Neuroscience 9, 12-17.

Chergui, K., Charlety, P.J., Akaoka, H., Saunier, C.F., Brunet, J.L., Buda, M., et al. 1993. Tonic activation of NMDA receptors causes spontaneous burst discharge of rat midbrain dopamine neurons in vivo. European Journal of Neuroscience 5, 137-144.

Conn, P.J., Pin, J.P., 1997. Pharmacology and functions of metabotropic glutamate receptors. Annual Reviews in Pharmacology and Toxicology 37, 205-237.

Fiorillo, C.D., Williams, J.T., 1998. Glutamate mediates an inhibitory postsynaptic potential in dopamine neurons. Nature 394, 78-82.

Freeman, A.S., Meltzer, L.T., Bunney, B.S., 1985. Firing properties of substantia nigra dopaminergic neurons in freely moving rats. Life Science 36, 1983-1994.

Gasparini, F., Lingenhohl, K., Stoehr, N., Flor, P.J., Heinrich, M., Vranesic, I., et al. 1999. 2-Methyl-6-(phenylethynyl)-pyridine (MPEP), a potent, selective and systemically active mGlu5 receptor antagonist. Neuropharmacology 38, 1493-1503.

Gonon, F.G., 1988. Nonlinear relationship between impulse flow and dopamine released by rat midbrain dopaminergic neurons as studied by in vivo electrochemistry. Neuroscience 24, 19-28.

Gonon, F.G., Buda, M.J., 1985. Regulation of dopamine release by impulse flow and by autoreceptors as studied by in vivo voltammetry in the rat striatum. Neuroscience 14, 765-774.

Grace, A.A., Bunney, B.S., 1984a. The control of firing pattern in nigral dopamine neurons: single spike firing. Journal of Neuroscience 4, 2866-2876.

Grace, A.A., Bunney, B.S., 1984b. The control of firing pattern in nigral dopamine neurons: burst firing. Journal of Neuroscience 4, 2877-2890.

Grace, A.A., Onn, S.P., 1989. Morphology and electrophysiological properties of immunocytochemically identified rat dopamine neurons recorded in vitro. Journal of Neuroscience 9, 3464-3481. 
Guatteo, E., Mercuri, N.B., Bernardi, G., Knopfel, T., 1999. Group I metabotropic glutamate receptors mediate an inward current in rat substantia nigra dopamine neurons that is independent from calcium mobilization. Journal of Neurophysiology 82, 1974-1981.

Horvitz, J.C., Stewart, T., Jacobs, B.L., 1997. Burst activity of ventral tegmental dopamine neurons is elicited by sensory stimuli in the awake cat. Brain Research 759, 251-258.

Ishii, T.M., Maylie, J., Adelman, J.P., 1997. Determinants of apamin and d-tubocurarine block in SK potassium channels. Journal of Biological Chemistry 272, 23195-23200.

Johnson, S.W., North, R.A., 1992. Two types of neurone in the rat ventral tegmental area and their synaptic inputs. Journal of Physiology 450, 455-468.

Johnson, S.W., Seutin, V., North, R.A., 1992. Burst firing in dopamine neurons induced by $\mathrm{N}$-methyl-D-aspartate: role of electrogenic sodium pump. Science 258, 665-667.

Kita, T., Kita, H., Kitai, S.T., 1986. Electrical membrane properties of rat substantia nigra compacta neurons in an in vitro slice preparation. Brain Research 372, 21-30.

Kitai, S.T., Shepard, P.D., Callaway, J.C., Scroggs, R., 1999. Afferent modulation of dopamine neuron firing patterns. Current Opinion in Neurobiology 9, 690-697.

Köhler, M., Hirschberg, B., Bond, C.T., Kinzie, J.M., Marrion, N.V. Maylie, J., et al. 1996. Small-conductance, calcium-activated potassium channels from mammalian brain. Science 273, 1709-1714.

Lacey, M.G., Mercuri, N.B., North, R.A., 1989. Two cell types in rat substantia nigra zona compacta distinguished by membrane properties and the action of dopamine and opioids. Journal of Neuroscience 9, 1233-1241.

Manley, L.D., Kuczenski, R., Segal, D.S., Young, S.J., Groves, P.M., 1992. Effects of frequency and pattern of medial forebrain bundle stimulation on caudate dialysate dopamine and serotonin. Journal of Neurochemistry 58, 1491-1498.

Meltzer, L.T., Serpa, K.A., Christoffersen, C.L., 1997. Metabotropic glutamate receptor-mediated inhibition and excitation of substantia nigra dopamine neurons. Synapse 26, 184-193.

Mercuri, N.B., Bonci, A., Calabresi, P., Stefani, A., Bernardi, G., 1995. Properties of the hyperpolarization-activated cation current $I_{h}$ in rat midbrain dopaminergic neurons. European Journal of Neuroscience 7, 462-469.

Mercuri, N.B., Stratta, F., Calabresi, P., Bernardi, G., 1992. A voltageclamp analysis of NMDA-induced responses on dopaminergic neurons of the rat substantia nigra zona compacta and ventral tegmental area. Brain Research 593, 51-56.

Mercuri, N.B., Stratta, F., Calabresi, P., Bonci, A., Bernardi, G., 1993. Activation of metabotropic glutamate receptors induces an inward current in rat dopamine mesencephalic neurons. Neuroscience 56, 399-407.

Mirenowicz, J., Schultz, W., 1996. Preferential activation of midbrain dopamine neurons by appetitive rather than aversive stimuli. Nature 379, 449-451.

Nissbrandt, H., Elverfors, A., Engberg, G., 1994. Pharmacologically induced cessation of burst activity in nigral dopamine neurons: significance for the terminal dopamine efflux. Synapse 17, 217-224.

Overton, P., Clark, D., 1997. Burst firing in midbrain dopaminergic neurons. Brain Research Reviews 25, 312-334.
Paladini, C.A., Fiorillo, C.D., Morikawa, H., Williams, J.T., 2001. Amphetamine selectively blocks inhibitory glutamate transmission in dopamine neurons. Nature, Neuroscience 4, 275-281.

Seutin, V., Johnson, S.W., North, R.A., 1993. Apamin increases NMDA-induced burst-firing of rat mesencephalic dopamine neurons. Brain Research 630, 341-344.

Shepard, P.D., Bunney, B.S., 1991. Repetitive firing properties of putative dopamine-containing neurons in vitro: regulation by an apamin-sensitive $\mathrm{Ca} 2^{+}$-acivated $\mathrm{K}^{+}$conductance. Experimental Brain Research 86, 141-150.

Shepard, P.D., German, D.C., 1988. Electrophysiological and pharmacological evidence for the existence of distinct subpopulations of nigrostriatal dopaminergic neuron in the rat. Neuroscience 27 , 537-546.

Shen, K.Z., Johnson, S.W., 1997. A slow excitatory postsynaptic current mediated by G-protein-coupled metabotropic glutamate receptors in rat ventral tegmental dopamine neurons. European Journal of Neuroscience 9, 48-54.

Shoepp, D.D., Goldsworthy, J., Johnson, B.G., Salhoff, C.R., Baker, S.R., 1994. 3,5-Dihydroxyphenylglycine is a highly selective agonist for phosphoinositide-linked metabotropic glutamate receptors in the rat hippocampus. Journal of Neurochemistry 63, 769-772.

Schultz, W., 1998. Predictive reward signal of dopamine neurons. Journal of Neurophysiology 80, 1-27.

Tong, Z.Y., Overton, P.G., Clark, D., 1996. Antagonism of NMDA receptors but not AMPA/kainate receptors blocks bursting in dopaminergic neurons induced by electrical stimulation of the prefrontal cortex. Journal of Neural Transmission 103, 889-904.

Tozzi, A., Guatteo, E., Caputi, L., Bernardi, G., Mercuri, N.B., 2001. Group I mGluRs coupled to $\mathrm{G}$ proteins are regulated by tyrosine kinase in dopamine neurons of the rat midbrain. Journal of Neurophysiology 85, 2490-2497.

Vezina, P., Kim, J.H., 1999. Metabotropic glutamate receptors and the generation of locomotor activity: interactions with midbrain dopamine. Neuroscience Biobehaviour Reviews 23, 577-589.

Wang, T., O'Connor, W.T., Ungerstedt, U., French, E.D., 1994. Nmethyl-D-aspartic acid biphasically regulates the biochemical and electrophysiological response of A10 dopamine neurons in the ventral tegmental area: in vivo microdialysis and in vitro electrophysiological studies. Brain Research 666, 255-262.

Wilson, C.J., Young, S.J., Groves, P.M., 1977. Statistical properties of neuronal spike trains in the substantia nigra: cell types and their interactions. Brain Research 136, 243-260.

Wu, Y.N., Johnson, S.W., 1996. Pharmacological characterization of inward current evoked by N-methyl-D-aspartate in dopamine neurons in the rat brain slice. Journal of Pharmacology and Experimental Therapeutics 279, 457-463.

Yung, W.H., Hausser, M.A., Jack, J.J.B., 1991. Electrophysiology of dopaminergic and non-dopaminergic neurons of the guinea-pig substantia nigra pars compacta in vitro. Journal of Physiology 436, 643-667.

Zheng, F., Gallagher, J.P., 1991. Trans-ACPD (trans-D,L-1-amino-1,3cyclopentanedicarboxylic acid) elicited oscillation of membrane potentials in rat dorsolateral septal nucleus neurons recorded intracellularly in vitro. Neuroscience Letters $125,147-150$. 